\title{
Structural effects of cap, crack and intrinsic curvature on the microtubule catastrophe kinetics
}

\author{
Cheng-Tai Lee and Eugene M. Terentjev ${ }^{1,}$ a) \\ Cavendish Laboratory, University of Cambridge, J.J. Thomson Avenue, Cambridge, CB3 OHE, \\ U.K.
}

Microtubule (MT) experience an effect called 'catastrophe', which is the transition from the MT growth to a sudden dramatic shrinkage in length. The straight GTP-tubulin cap at the filament tip, and the intrinsic curvature of GDP-tubulins are known to be the key thermodynamic factors that determine MT catastrophe, while the hydrolysis of this GTP-cap acts as the kinetic control of the process. Although several theoretical models have been developed, assuming the catastrophe occurs when the GTP-cap shrinks to a minimal stabilizing size, the structural effect of the GTP-cap and GDP-curvature was not explicitly included, thus their influence on catastrophe kinetics remains less understood. To investigate this structural effect, we apply a single-protofilament model with one GTP-cap while assuming a random hydrolysis mechanism, and take the occurrence of a crack in the lateral bonds between neighboring protofilaments as the onset of the catastrophe. Therein, we find the effective potential of the tip along the peel-off direction, and formulate the catastrophe kinetics as a mean first-passage time problem, subject to thermal fluctuations. We consider cases with and without a compressive force on the MT tip, both of which give a quadratic effective potential, making MT catastrophe an Ornstein-Uhlenbeck process in our formalism. In the free-standing case, the mean catastrophe time has a sensitive tubulin-concentration dependence, similar to a double-exponential function, and agrees well with experiment. For a compressed MT, we find a modified exponential function of force that shortens the catastrophe time.

\section{INTRODUCTION}

Microtubules (MT) are hollow cylindrical protein filaments composed of 10 to 17 parallel laterally-bonded protofilaments, the basic units of which are GTP-bound tubulin dimers that can later hydrolyze into its GDPform ${ }^{122}$. They are one of the key structural elements of the cell, and provide tracks for cargo transport with kinesin motors. MTs in vivo show a charateristic repeated dynamic pattern of slow growth and rapid shrinkage in length, a phenomenon termed as MT dynamic instability $\sqrt{3 / 4}$. In particular, the transition from the persistent MT growth to a rapid shrinkage is termed the MT 'catastrophe', while the reverse transition from the shrinkage back to growth is called the 'rescue ${ }^{46173}$. This dynamical instability of MTs offers an efficient way for re-organization of lonh filaments, e.g. at the onset of mitosis 78 , and an important 'search-and-find' mechanism to explore the cellular space and capture less diffusible cargoes for subsequent transport ${ }^{319}$ 11. It is therefore important to fully understand the underlying physical mechanisms behind this dynamic instability.

GTP-hydrolysis of tubulins is widely-accepted to be the main cause for MT instability 12 , in the sense that GDP-tubulins have an intrinsic curvature $\mathrm{e}^{1416}$ 19, while GTP-tubulins have a negligible curvature. Therefore, the natural hydrolysis can alter the conformation of the protofilament, which is initially rich in GTP-tubulins and has a naturally straight, to a protofilament rich in GDP-tubulins that has a naturally curled-up 'horn-like'

\footnotetext{
a) Electronic mail: emt1000@cam.ac.uk
}

structure 1420,22 , subsequently facilitating depolymerization of MT by lateral debonding 22 24. A few consecutive GTP-tubulins (known as the GTP-cap) at the growing MT tip are thought able to prevent the curling-up tendency of protofilaments in the MT, and hence inhibit the catastrophe $\overline{12|15| 23 \mid 25}$.

Accordingly, several theoretical models have been developed to investigate the dependence of MT catastrophe on free tubulin concentration in solution, focusing on kinetic evolution of the GTP-cap length through hydrolysis and tubulin-addition, and assuming that the catastrophe occurs when the cap shrinks below a certain minimal length required to maintain the MT stability 26 . These cap-models produced simple analytical expressions that could match with the MT catastrophe time, defined as the period of the MT growth phase, at varied tubulin concentrations in experiment. However, the length of this minimal stabilizing cap was either obtained by fitting experimental data, or pre-assumed to be one tubulin dimer, and hence was not justified from a microscopic view of MT structure. Moreover, these models do not account for the MT structural change induced by the change of the cap length over the evolution towards the catastrophe; its possible effect on the catastrophe time remains unclear, although the straight GTP-cap and the intrinsic curvature of GDP-tubulins have been known as a key thermodynamic factor in the catastrophe 1420 22 .

In this paper, we intend to explore this structural effect on MT kinetics, and will not apply the preassumption of a minimal stabilizing cap. We will focus on one protofilament of a whole MT, and assume this protofilament is composed of only two distinct regions: a GTP-tubulin cap (with zero intrinsic curvature), and the rest of it made of GDP-tubulins with a specific 


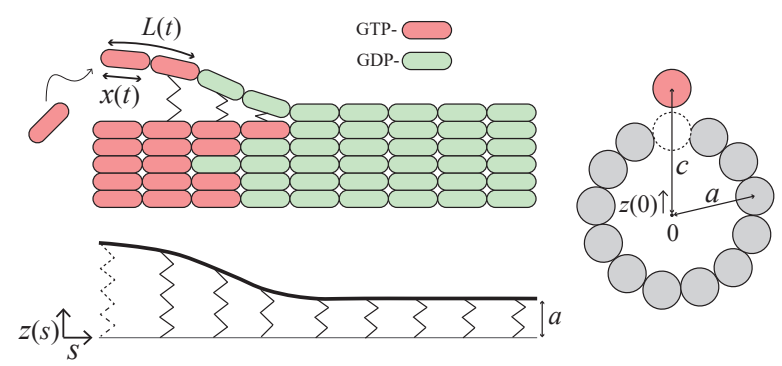

FIG. 1. A cartoon of a cylindrical MT filament composed of 13 protofilaments. Upper left shows the GTP-cap size $L(t)$, and the crack depth $x(t)$. Lower left: the lateral deflection $z(s)$ of the protofilament along the filament axis $s$. Right: the view along the longitudinal MT direction, showing the equilibrium position of the lateral effective-spring potential, $a$, and the bond-breaking distance, $c$.

natural curvature 16 , as shown in Fig. 1. The GTPtubulins in the cap region randomly hydrolyze into its GDP form ${ }^{3334}$, causing the cap length $L(t)$ to shrink, and eventually vanish if there is no tubulin addition on the tip.

Although random hydrolysis could produce GDPislands along the filament, we do not account for their possible influence on dynamics of MT catastrophe, because these islands are considered more important in the rescue event (shrinking MT reverting into the growing state 35 37. Besides, the cap at the tip is usually the longest stripe of consecutive GTP units. This is due to the fact that tubulins farther away from the addition site have spent longer in the assembly and are more likely to hydrolyze $\mathrm{e}^{38}$. The cap should therefore be the main contribution to structural change of the MT protofilament, and able to capture its effect on kinetics.

This one-protofilament model with one GTP-cap is undoubtedly a simplification of the real situation, where dynamics of other protofilaments in the same MT assembly may also contribute to the instability. Nevertheless, it produces acceptable theoretical results as shown in many previous studies $20126 / 29131 / 32$, and may act as a foundation to a more complicated model closer to the real situations 30 . In this paper, we will stay in this one-protofilament model, and will not further discuss the multi-protofilament case.

In our model, the protofilament is confined by the lateral bonds to neighboring protofilaments in its equilibrium radial position $(a)$, but is allowed to bend inward and outward subject to thermal fluctuations, with a chance to break the lateral bonds from the tip, if its radial position is stretched to a certain distance $(c)$. When a crack in the lateral bonds occurs, GTP-tubulin addition to the free-hanging cap is much slowed, and the protofilament is prone to quickly dissociate back into solution, due to lack of energy stabilization from lateral bonds $39 / 40$. Physically, this should initiate shrinkage of protofilament length (as dissociation of tubulins takes place rapidly in the peeled-off part, with only longitudinal bonds to be broken).

However, we are aware that the role of the crack on the MT catastrophe is still under debate in simulation studies. Margolin et al. pointed out that the depth of crack $x(t)$ may be an important factor in the initial catastrophe. In the growing phase, a short crack may exist, while an increase in crack may cause the catastrophe ${ }^{41 / 42}$. Later, $\mathrm{Li}$ et al. showed in simulation that the onset of catastrophe is determined by whether this crack reaches the GDP-region of the protofilament $(x(t) \rightarrow L)^{43}$. A more recent study further stated that the crack on itself is not sufficient to generate the instability, as the crack in a straight filament configuration is unstable and heals quickly; the crack has to couple with curled protrusion of protofilaments to cause the catastrophe ${ }^{22}$. Nevertheless, we want to point out that the molecular-dynamic simulation has a real difficulty dealing with the concept of mean first-passage time in stochastic processes (and generally, ensemble averages), which is what our work is about.

For the purpose of conveying a clear physical picture of the interplay between the GTP-cap and the crack in the catastrophe onset, we start form a simple model assuming that the occurrence of the crack does indicate an MT catastrophe. We will focus on how the structural change in protofilament induced by shrinkage of the cap affects the kinetics of producing such a crack - a protofilament with a short cap prefers to curl outward, and should therefore accelerate the peel-off. The time evolution of the cap length $L(t)$ and the crack depth $x(t)$ become the key to investigating the MT dynamics in our model.

The paper is structured as follows: in Sec. 2.1, we explain the kinetic equations of the crack depth $x(t)$ and the cap length $L(t)$, and their relation to the MT catastrophe. Sec. 2.2 introduces the method to calculate the effective potential for the protofilament tip to peel off and produce a crack in the lateral bonds. The tubulin unit at the tip undergoes a stochastic thermal motion in this potential, and we apply the MFPT method to estimate the average peel-off rate and hence the mean catastrophe time in Sec. 2.3. We fit the experimental data from the literature in Sec. 3, by using the mean catastrophe time we just obtained. Sec. 4 makes detailed comparisons with the previous cap models, and specifically investigates the effect of the intrinsic curvature. Finally, in Sec. 5 , we apply the similar procedures to find the mean catastrophe time when a compressive force is applied to the MT tip (e.g. when the MT is forced into a 'wall'), and extracting the force effect on the kinetics as an exponential function with a modification factor.

\section{THEORY}

\subsection{MT dynamic instability}

We assume that the cap length $L(t)$ increases when a GTP-tubulin (of length $b$ ) adds to the tip through a first- 


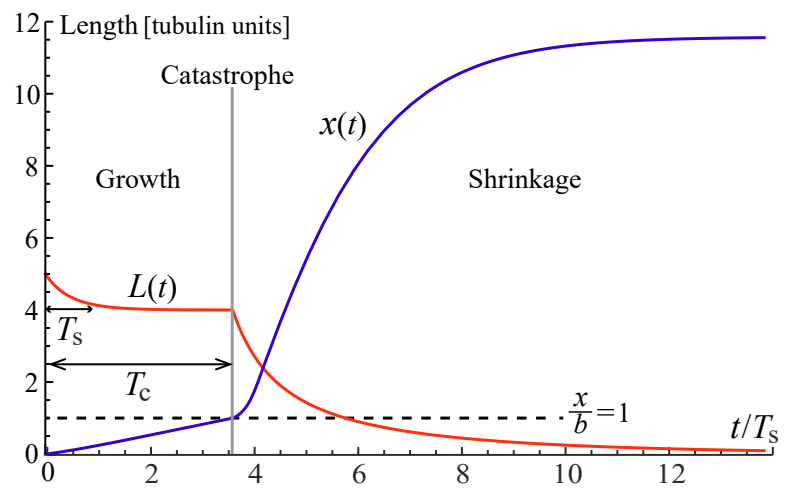

FIG. 2. An illustration of time evolution of the cap size $L(t)$ and the crack depth $x(t)$ in MT dynamics, obtained by solving Eqs. (1) with the dependence of $\left\langle k_{c}[L(t)]\right\rangle$ from the later part of the paper. The catastrophe begins when $x \geq b$ in our present model. $T_{s}$ is the time for the cap to reach its steadystate size, while $T_{c}$ is the catastrophe time.

order reaction, with a rate $b\left(k_{a} C\right)$, which is proportional to the free tubulin concentration $C$ in the solution. We also assume a large reservoir, so $C$ remains a constant in the course of MT dynamics. $L(t)$ decreases through random hydrolysis of GTP along the cap, which occurs with a probability density $k_{h}$ in time for each tubulin dimer $\left(k_{h}\right.$ is also known as the hydrolysis rate). To keep the model tractable, we do not consider the effect of the neighboring units on the hydrolysis rate ${ }^{31}$ nor the vectorial hydrolysis induced at the interface between the cap and the GDP-regime ${ }^{26}$. The average rate of cap shrinkage in this process can be estimated by the first moment: $-\sum_{m=1}^{L / b} m b k_{h}=-L(b+L) k_{h} / 2 b$. Another possible kinetic process to shrink the cap is the dissociation of a GTP-tubulin at the tip, breaking the longitudinal and lateral bonds at the same time. We think this process is rare from the thermodynamic viewpoint, while a number of experiments $6 \sqrt{6445}$ gave debatable results. For example, Drechsel et al $\stackrel{44}{ }$ extrapolated the experiment data and obtained an extremely small dissociation rate compared with the addition rate. On the other side of the spectrum, Walker et al ${ }^{[6}$ obtained a noticeable dissociation rate (still lower than the addition rate $k_{a}$ ). We will write our evolution equation for the GTP-cap length more generally, using the effective growth rate of the cap which is defined as: $b \tilde{k}_{g} \equiv b\left(k_{a} C-k_{T d}\right)$, with $k_{T d}$ the rate to dissociate one GTP-tubulin from the tip (by breaking both longitudinal and lateral bonds) during the MT growth phase. This rate $k_{T d}$ is independent of the free tubulin concentration $C$. When the crack appears $(x>b$, i.e. one full tubulin unit peels off), the tubulin addition stops and the cap grows no more.

On the other hand, the breaking of the lateral bonds, and thus creating of a crack, is a stochastic process: it is driven by thermal fluctuations and could occur at very different times - in this paper we analyze the distribution of these breaking times, or in other words, the mean firstpassage problem leading to an an average rate of peel-off $\left\langle k_{c}\right\rangle$. We will calculate this average rate in Sec. 2.3 For now, we only need to notice that $\left\langle k_{c}\right\rangle$ depends on the cap length $L(t)$. We further assume random dissociation of tubulin dimers along the peeled-off region (breaking the longitudinal bonds only), with a rate $k_{d}$ for each dimer. Similarly to the way we calculated the average shrinkage rate of the cap, $x(t)$ decreases with an average rate: $-x(b+x) k_{d} / 2 b$. Note that we do not include the rescue events, as its mechanism is still not fully understood and could possibly involve microtubule-associated proteins ${ }^{46 / 47}$, which is beyond the topic of this paper. Overall, two coupled kinetic equations for $L(t)$ and $x(t)$ take the form:

$$
\begin{aligned}
& \frac{d L(t)}{d t}=b \tilde{k}_{g} \Theta[x-b]-\frac{L(L+b)}{2 b} k_{h} ; \\
& \frac{d x(t)}{d t}=b\left\langle k_{c}[L(t)]\right\rangle-\frac{x(x+b)}{2 b} k_{d}\{1-\Theta[x-b]\},
\end{aligned}
$$

where the effective growth rate is defined including the possible tip-dissociation: $b \tilde{k}_{g} \equiv b\left(k_{a} C-k_{T d}\right)$, and $\Theta[x-b]$ is a step function: $\Theta=0$ when $x-b>0$, or $\Theta=1$ otherwise. We stress again: $\left\langle k_{c}\right\rangle$ is the average rate of peel-off, and is a function of the cap length $L(t)$.

In Fig. 2 we illustrate the characteristic dynamics of the growing and shrinking stages of MT, and also label the important time-scales involved in MT kinetics before catastrophe, through the numerical solutions for $L(t)$ and $x(t)$ of simultaneous Eqs. (1). The characteristic time $T_{s}$ is required to reach the steady state of $L$, while $\left\langle T_{c}\right\rangle$ is the average time for the catastrophe to occur. Figure 2 implies that the cap length in the MT has reached its steady-state value first, i.e. $T_{s}<\left\langle T_{c}\right\rangle$. In order to estimate $T_{s}$, we may first ignore the stopping of tubulinaddition due to the crack $(\Theta[x-b]$ is replaced with one in the equations), and solve for $L(t)$ :

$$
L(t)=\frac{b}{2}\left[-1+\sqrt{1+8 \frac{\tilde{k}_{g}}{k_{h}}}\left(1-\frac{2}{1+A_{0} e^{t / T_{s}}}\right)\right],
$$

where $A_{0}$ is a constant that needs to be determined from the initial condition $L(0)=L_{0} . A_{0}$ and $T_{S}$ take the form:

$$
\begin{aligned}
A_{0} & =\frac{2}{1-\left(\frac{L_{0}}{b}+\frac{1}{2}\right) \sqrt{\frac{k_{h}}{2 \tilde{k}_{g}+k_{h} / 4}}}-1 ; \\
T_{s} & =\frac{1}{\sqrt{2 k_{h}\left(\tilde{k}_{g}+k_{h} / 8\right)}} .
\end{aligned}
$$

The first branch of the contracting cap length $L(t)$ in Fig. 2 before the notional catastrophe that starts at $x(t)=b$, is exactly the Eq. (2). Note that to plot the numerical solution of the kinetic equations (1) we use the non-dimensional time axis scaled by $T_{s}$.

When $t \geq T_{s}, L(t)$ reaches the steady-state length $L_{s}$, given by:

$$
L_{s}=\frac{b}{2}\left(-1+\sqrt{1+8 \frac{\tilde{k}_{g}}{k_{h}}}\right) .
$$


At high tubulin concentration, Eq. (4) shows the squareroot scaling of $L_{s}$ with the ratio of two rates, $\left(\tilde{k}_{g} / k_{h}\right)$, as indeed seen in the earlier cap model\$26129131, while at zero tubulin concentration, $L_{s}$ vanishes, meaning that no steady cap remains.

On the other side, $\left\langle T_{c}\right\rangle$ is solved by integrating the growing crack from $t=0$ to $\left\langle T_{c}\right\rangle$, in the regime $x \leq b$ (without dissociation of tubulins, i.e. keeping $\Theta=1$ ): $\int_{0}^{\left\langle T_{c}\right\rangle}\left\langle k_{c}\right\rangle d t=1$. This integral equation is generally non-trivial, because $\left\langle k_{c}\right\rangle$ is a function of $L(t)$, and depends on time and the choice of initial cap length $L_{0}$. Nevertheless, if $\left\langle T_{c}\right\rangle \gg T_{s}$, it is plausible to remove such time-dependence in $\left\langle k_{c}\right\rangle$, by simply ignoring the small contribution before $t \leq T_{s}$ in the crack kinetics, and using the average peel-off rate $\left\langle k_{c s}\right\rangle$ estimated with the steady-state cap size $L_{s}$. Then the estimate is simple:

$$
\left\langle T_{c}\right\rangle \approx \frac{1}{\left\langle k_{c s}\right\rangle}
$$

This estimate may no longer be valid at a very low rate of tubulin addition $k_{a} C$ (i.e. $\tilde{k}_{q}$ becomes small), when the time $T_{s}$ becomes long, in which case only the numerical estimate of $\left\langle T_{c}\right\rangle$ is possible. In this section, we have linked the development of a crack in the lateral bonds with the MT catastrophe, and identified the mean catastrophe time $\left\langle T_{c}\right\rangle$. However, this equation (5) requires the explicit expression for $\left\langle k_{c}\right\rangle$, and its dependence on $L(t)$, which is our focus in the next section. Later, we will show that the MT kinetics is indeed in the regime $\left\langle T_{c}\right\rangle \geq T_{s}$ by comparing with experimental data on catastrophe times, and therefore Eq. (5) is applicable.

\subsection{Effective potential of tip curling}

We formulate the peel-off process in two dimension as depicted in Fig. 1, with the deflection in the radial direction $z(s)$ a function of position $s$ along the filament axis, ignoring any out-of-plane torsional effects. This protofilament has the bending rigidity $B$, with a GTPcap of length $L$ at the tip and the rest being homogeneously GDP-units. Following the established structural rules, we assume that GDP-units have a natural curvature $\kappa$, while GTP-units are straight. The lateral bonds from neighboring protofilaments are treated as an effective spring potential, with the elastic constant per length $k$, and the minimum at $z=a$. That is, $a$ is the equilibrium radial position for the straight GTP-protofilament, while choosing $z=0$ in the center of the MT cylinder. Altogether, the Hamiltonian for this protofilament is a sum of curvature and bonding terms, in the two regions:

$$
\begin{aligned}
H & =\int_{0}^{L} \frac{B}{2}\left(\frac{d^{2} z}{d s^{2}}\right)^{2}+\frac{k}{2}(z-a)^{2} d s \\
& +\int_{L}^{\infty} \frac{B}{2}\left(\frac{d^{2} z}{d s^{2}}-\kappa\right)^{2}+\frac{k}{2}(z-a)^{2} d s
\end{aligned}
$$

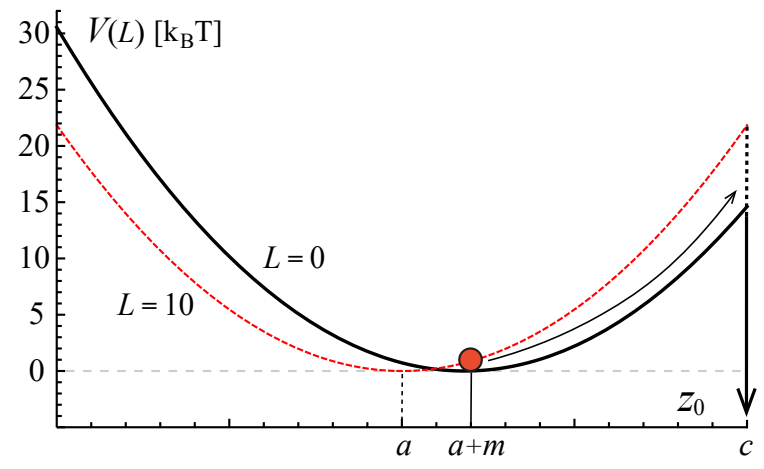

FIG. 3. Illustration of the MFPT problem in the quadratic effective potential $V$, a function of the cap size $L$. For a long cap size $L=10$ (tubulin units), the potential minimum is almost at $z_{0}=a$, while without the cap $L=0$, this minimum is shifted to $(a+m)$, approaching the bond-breaking distance $c$ (i.e. the adsorbing boundary in our model). The tip, shown as the red circle, initially starts at the potential minimum, and has a chance to reach $z_{0}=c$, subject to thermal fluctuations.

The first line is for the GTP-cap, and the second is for the long GDP part of the protofilament.

To find the average peeling rate $\left\langle k_{c}\right\rangle$, we need to determine the effective potential for the unit at the filament tip, along the coordinate $z(s=0)$. In fact, the search for this potential has been carried out by Jánosi et al. ${ }^{[20}$ Yet, they offered only the qualitative discussion of the structural role of the GTP-cap in the MT catastrophe, and did not investigate how hydrolysis and tubulin-addition rate would involve in this kinetic process. We will use the effective potential found in Ref ${ }^{[20}$ to calculate $\left\langle k_{c}\right\rangle$ using the MFPT method, and combine it with Eq. (5). We briefly review their method below.

The idea is to find the static configuration for $z(s)$ with the tip held at position $z(0)=z_{0}$, along with some other boundary conditions to be specified, and then insert this static solution back into the Hamiltonian functional of Eq. (6), obtaining $H$ as a function of $z_{0}$. The static equation for $z(s)$ in the Hamiltonian functional of Eq. (6) has the same form for both the cap and the GDP-part:

$$
B \frac{d^{4} z}{d s^{4}}+k(z-a)=0
$$

In principle, the exact static solution for $z(s)$ is a piecewise function connected at the interface $s=L$, satisfying two boundary conditions at each end and four more at the interface. However, this exact method results in an undesirable expression for the solution, and the effective potential subsequently obtained is complicated, preventing clear interpretation for the key physical factors underlying the peel-off process.

This complexity may be avoided by using the Rayleigh-

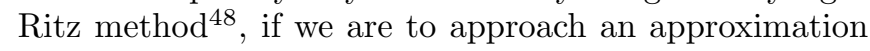
for the static shape and then for its energy - proposing an ansatz for the static solution, with one parameter to be determined by minimizing the Hamiltonian (6) 
when inserted back into this equation. Respecting local force balance along the protofilament, it is natural to choose this ansatz to be the general solution of Eq. (7) that meets boundary conditions at both ends $z(0)=z_{0}$, $d^{2} z /\left.d s^{2}\right|_{s=0}=0, z(\infty)=a$ and $d z /\left.d s\right|_{s=\infty}=0$, while ignoring the small deviation that would be produced at interface $s=L$ :

$$
z(s)=a+\left[\left(z_{0}-a\right) \cos \left(\frac{s}{\lambda}\right)+A \sin \left(\frac{s}{\lambda}\right)\right] e^{-s / \lambda},
$$

where $\lambda=(4 B / k)^{1 / 4}$, and the amplitude $A$ is a parameter determined through energy minimization $d H / d A=0$, giving:

$$
A=-\kappa \sqrt{\frac{2 B}{k}} \cos \left(\frac{b L}{\lambda}+\frac{\pi}{4}\right) e^{-b L / \lambda} .
$$

The static protofilament shape is, in essence, an oscillating exponential decay function of the filament coordinate $s$. The decay length $\lambda$ entails a stronger curling-up effect in a more rigid protofilament (higher $B$ ), while the strength of lateral bonds $k$ plays the reverse role, trying to hold the protofilament onto the straight configuration.

Inserting the solution of Eqs. (8) and (9) back into the energy functional (6), we obtain the effective potential $V$ as a function of the tip height $z_{0}$ :

$$
\begin{gathered}
V\left(z_{0}\right)=\frac{B^{\frac{1}{4}} k^{\frac{3}{4}}}{2 \sqrt{2}}\left(z_{0}-a-m\right)^{2}, \\
\text { with } m(t)=\kappa \sqrt{\frac{2 B}{k}} \cos \left(\frac{L}{\lambda}-\frac{\pi}{4}\right) e^{-L / \lambda} .
\end{gathered}
$$

Equation 10 in the form of a quadratic potential is the key result in Jánosi et al.20. As the cap length $L$ decreases with time, the natural curvature $\kappa$ of GDP units shifts this minimum to a larger value, $(a+m)$, with $m$ increasing and eventually reaching $m=\kappa \sqrt{B / k}$ for zero GTP-cap. On the other hand, $m$ vanishes when the GTP cap is long, recovering the straight protofilament. It is interesting to notice that $\kappa$ has no effect on the restoring force, which is only determined by the bending rigidity of the protofilament and the neighboring lateral bonds.

The change in the natural curvature $\kappa$ may shift the potential well $z_{0}=a$ in a totally different trend, depending on the $L$-value (making cosine-function positive or negative). For a small cap length $L$, the potential minimum is gradually shifted outward as $\kappa$ increases (due to a positive $m$ ), while a longer cap size has the opposite effect. The cross-over can be readily obtained by solving the condition to make the cosine-function in $m$ negative: $L^{*} / \lambda-\pi / 4=\pi / 2$, giving $L^{*}=3 \lambda / 4 \pi$. We will explore how this somehow unintuitive phenomenon will affect the catastrophe time in Sec. 4 below. With this effective potential, we are now able to solve the stochastic problem and find $\left\langle T_{c}\right\rangle$ in the next section.

\subsection{MFPT of the tip peeling off}

In our model, when the tip tubulin unit is stretched to $z_{0}=c$ in the course of its thermal fluctuations, the lateral bonds break off, producing a crack and initiating the MT catastrophe. We cast this kinetic process of escaping from a quadratic potential (also known as the Ornstein-Uhlenbeck process) into a standard onedimensional MFPT problem $\stackrel{49150}{ }$, with the adsorbing boundary condition at $z_{0}=c$, and a reflective condition at $z_{0}=-a$ where the protofilament hits the opposite wall of the MT cylinder. We let the tip initially start from the potential well $z_{0}=(a+m)$, instead of using an equilibrated probability, respecting the fact that the restoring constant of this potential is strong in the MT case, and the tip stays at the bottom of this well most of the time (thus a good approximation). The illustration for this process is plotted in Fig. 3, using the parameter values we will find in the later Sec. 3

Note that the cap size $L$ is evolving with time, due to tubulin-addition at the front, and GTP-hydrolysis at the back of the cap, as shown in Eq. (2), essentially making the effective potential $V(L)$ also time-dependent. If the time-scale for this potential to reach its steady state is much shorter than the average peel-off time, namely, $\left\langle T_{c}\right\rangle \gg T_{s}$, one can safely adopt the time-independent steady-state potential to formulate the MFPT problem. In our case, it means substituting $L_{s}$ of Eq. (4) for $L$ in Eq. (10). The MFPT for such a time-independent potential, with adsorbing and reflective boundaries at $z_{0}=c$ and $-a$, respectively, is a well-known integral expression $\frac{51152}{}$ :

$$
\langle\tau\rangle=\frac{1}{D} \int_{a+m}^{c} e^{\beta V\left(z_{0}\right)}\left(\int_{-a}^{z_{0}} e^{-\beta V(y)} d y\right) d z_{0},
$$

where $D$ is the diffusion coefficient for the radial fluctuations of the tubulin dimer at the tip, and $\beta=1 / \mathrm{k}_{\mathrm{B}} \mathrm{T}$ is the inverse thermal energy.

On the other hand, if the cap changes slowly with time, the time-dependence in $V$ though $L(t)$ cannot be ignored, making Eq. (11) inapplicable (the time translational invariance used in deriving this integral expression is broken in this case, and the backward diffusion operator becomes non-trivia[52). A possible strategy to tackle MFPT problems (or Kramer kinetics theory) with a time-dependent potential was proposed in the context of non-equilibrium single molecule pulling experiments 53 .

However, in MT catastrophe, a most probable (steadystate) cap size larger than one tubulin unit is frequently observed ${ }^{54} \frac{56}{2}$, so it is not unreasonable to assume the steady-state effective potential, in order to find out $\langle\tau\rangle$, then use this result to fit experiment data, and then return to justify the assumption $\langle\tau\rangle \gg T_{s}$. Under this scenario, Eq. (5) gives $\left\langle T_{c}\right\rangle=\langle\tau\rangle$, by noticing $\left\langle k_{c s}\right\rangle=1 /\langle\tau\rangle$.

Assuming a strong restoring constant for the harmonic potential, and staying in the regime where the position of the potential minimum never passes beyond the bondbreaking distance $c$, we combine Eqs. (10) and (11) to 
obtain 57 :

$$
\left\langle T_{c}\right\rangle=\frac{\sqrt[4]{32 \pi^{2}} \operatorname{Exp}\left[\frac{B^{1 / 4} k^{3 / 4}}{2 \sqrt{2}} \beta\left(c-a-m_{s}\right)^{2}\right]}{D \beta^{3 / 2} B^{3 / 8} k^{9 / 8}\left(c-a-m_{s}\right)},
$$

where $m_{s}$ is the shift due to interplay between the steady-state cap and the natural curvature $\kappa$ of GDPunits, and is given in Eq. (10) with $L(t)$ replaced by the concentration-dependent steady value $L_{s}$ of Eq. (4) there:

$$
\begin{gathered}
m_{s}=\kappa \sqrt{\frac{2 B}{k}} \cos \left[\frac{b}{2 \lambda}\left(-1+\sqrt{8 \frac{\tilde{k}_{g}}{k_{h}}+1}\right)-\frac{\pi}{4}\right] \\
\times \operatorname{Exp}\left[\frac{-b}{2 \lambda}\left(-1+\sqrt{8 \frac{\tilde{k}_{g}}{k_{h}}+1}\right)\right] .
\end{gathered}
$$

The length scale $\lambda=(4 B / k)^{1 / 4}$, is the same as given below Eq. (8). As tubulin concentration $C$ decreases, the cap is shrinking due to a decreased effective grwoth rate $\tilde{k}_{g}$, causing $m_{s}$ to approach the value $\kappa(2 B / k)^{1 / 2}$; with this, the catastrophe time gradually reduces to the case of a fully-curved protofilament (composed of all GDP-units at zero tubulin concentration in solution). At high tubulin concentration $C$, it is expected that the cap length is long, and hence the catastrophe time should eventually resemble the case of a fully-straight protofilament. This can be examined by noticing $m_{s} \approx 0$ at large $C$.

The influence of the natural curvature $\kappa$ is manifested in the exponent of Eq. 12 through $m_{s}$ (and also as a minor factor in the denominator), implying that catastrophe time should be sensitive to the change in intrinsic curvature. And this curvature-dependence should become more pronounced as $C$ decreases, as can be seen from the expression for $m_{s}$ of Eq. (13) (the coefficient after $\kappa$ decays fast as $C$ increases and $k_{g}$ accordingly becomes larger). However, in practice, one has to assume the natural curvature of the GDP-tubulin is a fixed value determined by structural analysis. We now proceed to find reasonable values for parameters used in the mean catastrophe time $\left\langle T_{c}\right\rangle$ of Eq. 12.

\section{CATASTROPHE OF A FREE-STANDING MT}

The frequency of MT catastrophes is the commonlymeasured quantity in experiment; in our model it is defined as the inverse of the peel-off time, $1 /\left\langle T_{c}\right\rangle$, because catastrophe is assumed to occur when the actual crack in lateral bonds first appears $(x \geq b$ in Fig. 2). We can now test the validity of Eq. (12) by fitting the catastrophe frequency with the data from Drechsel et al! ${ }^{44}$, at varied growth rates $\tilde{k}_{g}$. Parameters are specified as follows: the bond-breaking distance $(a-c)$ is $8 \mathrm{~nm}$ (roughly the length of one tubulin unit), as suggested by nano-indentation experiments on MT filament Each GDP-unit roughly curls up to 0.21 radian $14|60| 61$, giving the curvature $\kappa=0.21 / 8 \sim 0.026 \mathrm{~nm}^{-1}$. The value of bending rigidity $B$ of a single protofilament is still under debate, varying form 1500 to $13000 \mathrm{pN} \cdot \mathrm{nm}^{2}$ in different reports (corresponding to a straightening energy of a GDP-unit between 3.7 and $32 \mathrm{k}_{\mathrm{B}} \mathrm{T}$ at room temperature $)^{62} 65$. Here, we choose a soft rigidity limit that gives the persistence length of 50 tubulin units at room temperature (equally, $B \sim 1645.6 \mathrm{pN} \cdot \mathrm{nm}^{2}$ ).

The diffusion coefficient $D$ of a tubulin dimer is constrained between the measurements of $0.6 \cdot 10^{-11}$, in vivo, and $5.5 \cdot 10^{-11} \mathrm{~m}^{2} / \mathrm{sec}$, in vitrd 6 66]67. The rate of hydrolysis $k_{h}$ was reported to have values below 1 tubulin/sec ${ }^{29168}$, however, we keep $k_{h}$ and the elastic constant $k$ are our fitting parameters. Figure 4 shows the plot of mean catastrophe frequency $1 /\left\langle T_{c}\right\rangle$ from Eq. (12), with the points being the experimental data from Drechsel et al. ${ }^{44}$, for the increasing effective growth rate of the GTP-cap $\tilde{k}_{g}$ (or simply put, the increasing rate of tubulin addition). The result of fitting gives: $k_{h}=0.1536 \mathrm{sec}^{-1}$, $D=0.64 \cdot 10^{-11} \mathrm{~m}^{2} / \mathrm{sec}$ : a smaller diffusive mobility, as, in fact, expected because the experiment was conducted in vivo, and $k=5.32 \cdot 10^{5} \mathrm{~N} / \mathrm{m}^{3}$, corresponding to $33.16 \mathrm{k}_{\mathrm{B}} \mathrm{T}$ at room temperature to break the neighboring lateral bonds, estimated from the expression $k b(c-a)^{2} / 2$. This $k$-value is consistent with the estimated dissociation energy for one lateral bond in a number of previous studies $2159163164 \mid 69$, which ranged from 3 to $15 \mathrm{k}_{\mathrm{B}} \mathrm{T}$, corresponding to 6 to $30 \mathrm{k}_{\mathrm{B}} \mathrm{T}$ in our model (as there are actually two neighboring protofilaments, thus two lateral bonds to break). Note that the lower estimated lateral bond energy is obtained through interpolating kinetic equations; it will undoubtedly also depend on how well the proposed kinetic model connects with the real reaction mechanism, while direct indentation experiments favors a larger value, which our fitted $k$ also does. By using Eq. (4) with the fitted hydrolysis rate constant $k_{h}=0.154 \mathrm{sec}^{-1}$ inserted, the steady-state cap size $L_{s}$ is estimated to increase from 2 to 5 tubulins, when the effective growth rate $\tilde{k}_{g}$ varies from 0.46 to 2.3 tubulins/sec, covering the experiment range of Drescher et al ${ }^{44}$ (red solid circles in Fig. (4). The cap size at the medium and low concentration regimes indeed remains small.

Our proposed formula for $\left\langle T_{c}\right\rangle$, Eq. (12), is clearly compatible with experimental data. Due to the cosinefunction embedded in $m_{s}$, the expression for $\left\langle T_{c}\right\rangle$ shows a characteristic non-monotonic change at the medium effective growth rate. As the cap growth rate increases, $\left\langle T_{c}\right\rangle$ eventually reaches the steady-state limit of a long GTP-cap (the straight protofilament case), where $m_{s}$ in $\left\langle T_{c}\right\rangle$ of Eq. (12) becomes zero. This asymptotic plateau is shown as the grey dashed line in Fig. 4. We will discuss this plateau at high tubulin concentration in the next section.

With the fitted hydrolysis rate, we are now able to examine the assumption $\left\langle T_{c}\right\rangle \gg T_{s}$, which we have adopted in the derivation. At zero growth rate $\tilde{k}_{g}=0$, we obtain $T_{s} \approx 13 \mathrm{sec}$. As the growth rate increases, $T_{s}$ gradually decreases roughly with the scaling $\left(\tilde{k}_{g}\right)^{-1 / 2}$, eventually intercepting the increasing curve for $\left\langle T_{c}\right\rangle$; this crossover 


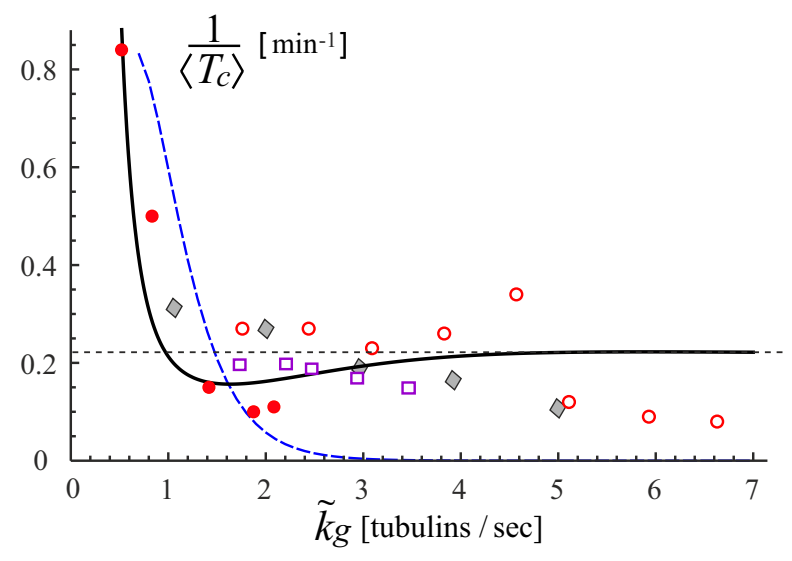

FIG. 4. Catastrophe frequency at varied effective growth rate of the GTP-cap (which grows with the free tubulin concentration: $\left.\tilde{k}_{g}=b\left(k_{a} C-k_{T d}\right)\right)$. The solid black line is plotted using Eq. 12 , with the parameter values obtained by fitting the data from Drescher et al ${ }^{44}$ (red solid circles). The asymptotic plateau at high growth rate is obtained by by letting $m_{s}=0$ in Eq. 12, and is the peel-off rate at the tip for a fully-straight protofilament. The blue dashed line accounts for the effect of crack-healing, with the parameters values obtained by re-fitting the same data (the resultant parameter values are slightly different from the black solid curve). Gray diamonds ${ }^{73}$, purple squares ${ }^{70}$ and red open circles ${ }^{6]}$ show the catastrophe frequency at different growth rates in various other experiments.

occurs around $\tilde{k}_{g}=0.2($ tubulin/sec), using the same parameter values as determined above. Note that the lowest experimental rate in Fig. 4 is $\tilde{k}_{g} \approx 0.52$. Therefore, the predicted value of $\tilde{k}_{g}$ at intersection is far below the practical experiment range. The assumption $\left\langle T_{c}\right\rangle \gg T_{s}$, and the Eq. (5), which we have used to find Eq. (12) are therefore valid.

\section{CRITICAL COMPARISON OF CAP MODELS}

Our main result, Eq. 12 for $\left\langle T_{c}\right\rangle$, has several features that were already observed in the previous studies. First, our formulation of $\left\langle T_{c}\right\rangle$ does not consider the fluctuation of the cap length, since we use the average cap length $L(t)$, unlike the classical work by Flyvbjerg et al.26, and some other similar cap-loss or shrinkage models 27/29131 which all assume that MT catastrophe is induced by cap length fluctuation (to a small cap size). Instead, we regard the occurrence of catastrophe as a thermallyinduced escape problem along the radial direction, from a quadratic effective potential, allowing us to capture the structural effect of the average cap length (which is proportional to $C^{-1 / 2}$ when the dissociation rate $k_{T d}$ is negligible), and the intrinsic curvature $\kappa$. This results in an exponential function with the exponent $C^{-1 / 2}$ in the potential, hence contributing to a something like a double exponential dependence on tubulin concentration in $\left\langle T_{c}\right\rangle$. In contrast, $T_{c}$ in previous cap models appears as a algebraic fraction of concentration:

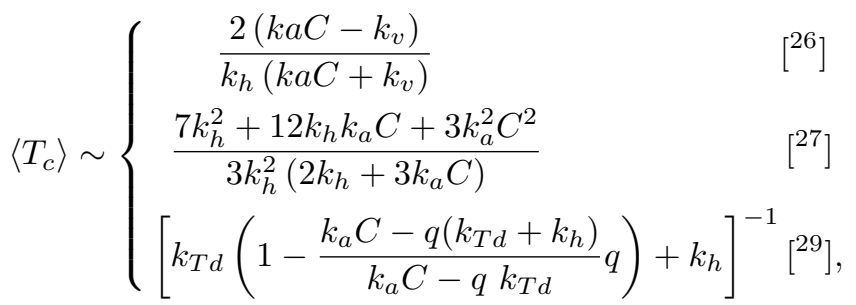

where $k_{v}$ and $k_{T d}$ are the vectorial hydrolysis rate (the rate at which the interface between the GTP-cap and GDP-part moves toward the GTP-tip), and the longitudinal dissociation rate of one GTP-tubulin dimer during the MT growing phase as defined earlier in Eq. (1), respectively. $q$ is the probability of having a GTP-tubulin at the tip. $q$ is, in fact, a function of tubulin concentration and other rate constants involved in this mechanism, yet no direct analytical expression of $q$ was given by Ranjith et al. ${ }^{[29}$ (only a set of kinetic equations required to solve $q$ ). As a result, the concentration dependence becomes somehow unstraightforward to grasp (but physically speaking, $q \approx 0$ and $\approx 1$ at very low and high tubulin concentrations, respectively, as stated in their supporting material). Note that Flyvbjerg's result ${ }^{26}$ was obtained by assuming the minimum stabilizing cap size being one unit, while Brun et al. ${ }^{27}$ obtained this size as two units by fitting experimental data. Ranjith's work ${ }^{29}$ was not limited to the stabilizing cap size of unity, and numerically extended to any cap size; here we listed only the analytical result corresponding to the case of the cap of one tubulin. This also highlights the issue, which was also pointed out in Ranjith's work ${ }^{29}$, that if the minimum stabilizing cap size is equal to one, then the catastrophe frequency saturates at a constatnt level at high tubulin concentrations: one must have this minumum size greater than one, as indeed in Brun et al.27.

As tubulin concentration decreases, $\left\langle T_{c}\right\rangle$ in Flyvbjerg's model decreases, and becomes zero at $k_{a} C=k_{v}$, while $\left\langle T_{c}\right\rangle$ reduces to $1 /\left(k_{T d}+k_{h}\right)$ in Ranjith's, and to $7 / 6 k_{h}$ in Brun's models, respectively. Although our $\left\langle T_{c}\right\rangle$ cannot be taken to very low concentration (because we require $\left\langle T_{c}\right\rangle \geq T_{s}$ to use the time-independent potential), the MFPT method we use will not give a zero $\left\langle T_{c}\right\rangle$ (diverging rate) either. It is because at zero concentration the most probable cap size $L_{s}$ should be zero, while any non-zero cap size $L(t)$, in the progress towards the steady-state $L_{s}=0$, should give a longer catastrophe time. Therefore $\left\langle T_{c}\right\rangle$ is bound by the shortest possible catastrophe time at $\tilde{k}_{g}=0$, and hence $m_{s}=\kappa(2 B / k)^{1 / 2}$ in Eq. $[12)$, in this low concentration limit. Note that the loss of the dependence of the hydrolysis rate $k_{h}$ in this zero concentration limit arises from the application of our ensemble-averaged cap size $L_{s}$, which is the only quantity that $k_{h}$ appears in our model. If one wishes to investigate the catastrophe time in one single MT filament in this low-concentration limit (instead of pursuing the ensemble behavior), the cap size $L_{s}$ may be set to one unit: the minimum cap size in the MT growth phase, in Eq. 12. As for Rangith's 
and Brun's27 results, their $\left(1 / k_{h}\right)$ dependence at $C \rightarrow 0$ results from the limitations of the method - they assume that the peel-off of protofilaments occurs fast after being fully hydrolyzed, and the longitudinal dissociation immediately follows, so the limiting catastrophe time will be solely proportional to the time required to hydrolyze that last GTP-unit.

At high concentration, our model will eventually reach a plateau frequency of catastrophe, similar to Flyvbjerg and Ranjith models with minimum cap size of one, with their asymptotic $T_{c}$ being $2 / k_{h}$ and $1 / k_{h}$, respectively. Nevertheless, the physical meanings of this plateau are quite different: our asymptotic expression represents the peel-off time at the tip of a fully-straight protofilament, with the hydrolysis having no effect in this limit. In contrast, the $k_{h}$ factor in these two models arises from the assumption that the catastrophe occurs when one GTPtubulin at the very tip is removed through hydrolysis (with the rate $k_{h}$ ). Note that the dissociation rate $k_{T d}$ depends on the experiment setting $g^{6 / 44 \mid 45}$, and can be almost zerd 44 . With zero $k_{T d}$, Ranjith's asymptotes (for the minimum $L_{s}=b$ ) at high and low concentrations give the same expression $\left(1 / k_{h}\right)$, which seems troublesome as one expects the catastrophe time to be far shorter at very low tubulin concentration. Indeed, Ranjith's work ${ }^{29}$ showed that taking the minimum stabilizing cap size to be two fitted the experiment data from Drechsel et al. $\underline{44}$ better. On the other side, Brun's expression for catastrophe rate diverges at high concentration $\left(T_{c} \approx k_{a} C / 3 k_{h}^{2}\right)$, reflecting the competition between hydrolysis and addition, when there are two GTP-units at the tip. When one GTP-tubulin has been hydrolyzed, the catastrophe will not yet occur, and an addition of a new GTP-unit to the tip will again make two-unit cap.

It is perhaps more intuitive to think that high tubulin concentration will result in persistent growth of MT, see the experiment data from Gardner et al. (purple squares ${ }^{70}$ and Walker et al. (red open circles ${ }^{\sqrt{6}}$ in Fig. 4. which appear to approach zero or a small value at high $\tilde{k}_{g}$. Then in theoretical expression, the catastrophe frequency should go towards zero (like in Brun's result), or at least a small value (like in Flyvbjerg's and Ranjith's expressions, although it is hard to understand how one single GDP-unit at the tip, followed by consecutive straight GTP-units at high tubulin concentration, could reproduce the experimentally observed horn-like shape of peeled MT protofilaments). Or the catastrophe frequency could have a cut-off point beyond which catastrophe does not occur. Our result presented in Fig. 4 seems not to produce this expected trend: the catastrophe frequency at high concentration is even slightly larger than in the medium range. Nevertheless, we must understand that our model is a minimal model emphasizing the structural effect of the tubulin concentrationrelated cap on the crack-producing of lateral bonds (and then the catastrophe kinetics). Some other possible kinetic processes may become important and suppress the catastrophe at very high concentrations.
For example, MD simulation of Zakharov et al ${ }^{22}$ pointed out that healing of the crack is fast for a filament in somewhat straight shape, and a curled protrusion of protofilaments is required to initiate the catastrophe. This may imply that healing of the crack may also be an important factor to fully describe the MT catastrophe dynamics in the high concentration regime, where crack-healing could be fast, due to a longer average GTPcap (causing a more straight configuration). This healing rate of a crack should also be a function of tubulin concentration (through varying GTP-cap, and the resultant protofilament structure) and the peeled-off length.

We attempted to capture this crack-healing effect by imposing a correction factor in front of the crackproducing rate $\left\langle k_{c}\right\rangle$. This factor may be estimated by the equilibrated probability for the laterally-debonded tubulin to stay away from the bond-breaking distance $c$ in the outward lateral direction, i.e. calculating the normalized partition function of the bending energy penalty to overcome in this case. Then, we used this modified cracking rate to re-fit the experiment data from Drechsel et al $\stackrel{44}{4}$, producing the blue dashed curve in Fig. 4 . Although this blue curve gives the 'expected' physical result, that $1 /\left\langle T_{c}\right\rangle$ approaches zero in the large- $k_{g}$ limit, its decay rate with increasing tubulin concentration (or equally, increasing $\tilde{k}_{g}$ ) drops to zero at a far too low concentration, inconsistently with the decaying trend given by experiment data presented in Fig. 4

Alternatively, we may consider another possible kinetic mechanism: once the crack occurs, the removal of laterally-debonded tubulin immediately follows, faster than the rate of re-attaching back to heal the crack. Under this scenario, the healing effect may be ignored, and the catastrophe frequency will have the trend as Eq. (12) (the black solid curve in Fig. (4) at low and medium growth rates $\tilde{k}_{g}$. Yet, at high growth rates, rescue events, where free GTP-tubulins add on to GTP-tubulin islands from the tip to stop further shrinkage of protofilament length ${ }^{35}$ 37, should become more frequent. In this sense, the rapid rescue at high growth rates (giving a very short shrinkage episodes) causes MTs to seemingly grow almost persistently - the shrinkage due to the crack-dissociation process is indistinguishable with the stochastic fluctuation in the MT length. So the experimental observation that the catastrophe frequency (slowly) goes to zero in this limit may not be incompatible with the plateau predicted byEq. (12).

We also notice that when $k_{h}=0$, the catastrophe time in Eq. 12 is non-zero, which is against many experiment reports $\$ 2123171$. However, this is a more or less similar issue we have discussed at high growth rates $\tilde{k}_{g}$. As $k_{h}$ decreases, it is expected that the GTP-islands along the protofilament become larger and longer, facilitating MT rescue events. Without hydrolysis $\left(k_{h}=0\right)$, each time adding one tubulin to the protofilament tip, it successfully attaches to GTP-islands, and hence rescue occurs. Consequently, even though the crack appears and dissociation of one debonded tubulin does occur, MT protofila- 

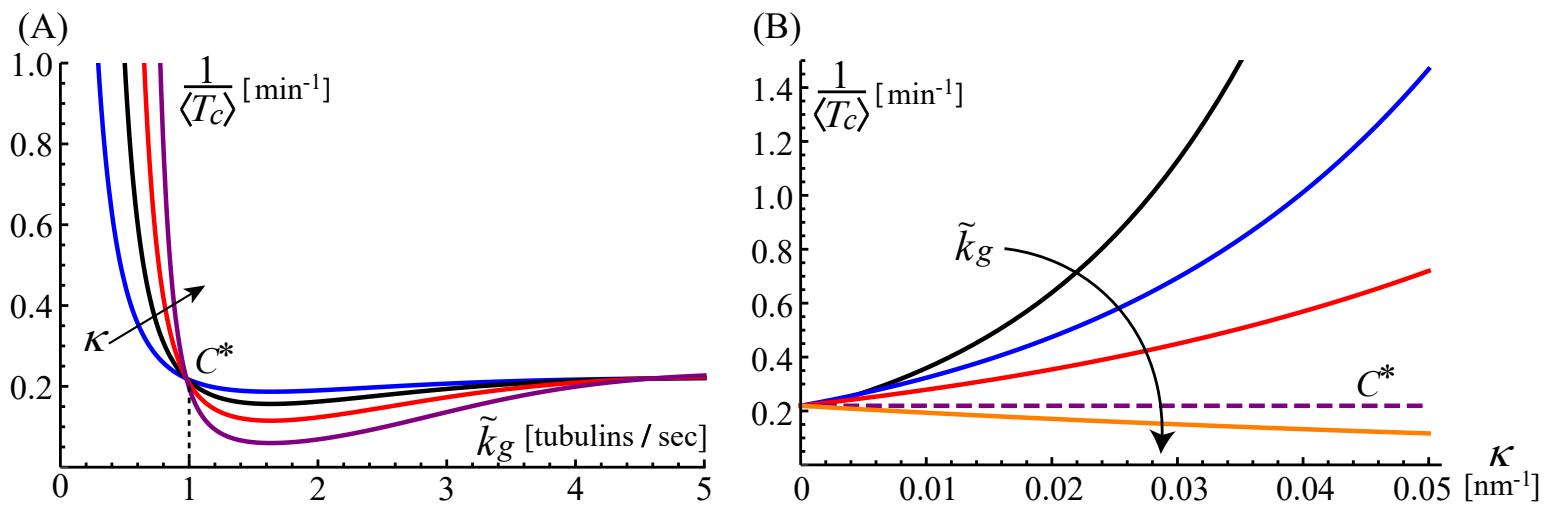

FIG. 5. Catastrophe frequency $1 /\left\langle T_{c}\right\rangle$ plotted against $(\mathrm{A})$ effective growth rate of the GTP-cap $\tilde{k}_{g}$, with intrinsic curvature $\kappa=0.0125,0.026,0.05$ and $0.1 \mathrm{~nm}^{-1}$, increased along the arrow. (B) intrinsic curvature $\kappa$, with $\tilde{k}_{g}=0.5,0.6,0.7,0.98$ and 1.5 tubulins/sec, increased along the arrow. $C^{*}$, shown by dashed lines in both plots, is the characteristic tubulin concentration, at which $\kappa$ has no effect on $T_{c}$, and $C^{*}$ separates out two regimes with opposite $\kappa$ effects on $T_{c}$.

ments are immediately 'rescued', resulting in an appearance of persistent growth (as in the case with high $\tilde{k}_{g}$ above). Our model here only focuses on catastrophe events, and does not explore the relation between MT rescue frequency and tubulin concentration or hydrolysis rate. Therefore, the theory presented here is yet incomplete to fully describe the MT dynamics, but serves as a starting point to investigate MT dynamics from a structural (mechanical) and thermodynamic viewpoint.

Our model explicitly and quantitatively addresses the effect of natural curvature $\kappa$ on the MT catastrophe time through Eq. (12), which previous theories did not explore. We plot the catastrophe frequency against effective growth rate $\tilde{k}_{g}$ for varied intrinsic curvature $\kappa$ in Fig. 5(A), with all other parameter values the same as used in producing the black solid line of Fig. 4 , see also the descriptions on fitted parameters in Sec. 3. The effect of natural curvature $\kappa$ on the mean catastrophe time $\left\langle T_{c}\right\rangle$ is very different in the two concentration regimes separated by a characteristic cap size $L^{*}$ and hence a concentration $C^{*}$ where $m_{s}$ changes signs from positive to negative (due to its cosine-function) in Eq. 13):

$$
L^{*}=\frac{3 \lambda}{4} ; C^{*}=\frac{1}{k_{a}}\left[k_{T d}+\frac{3 \pi k_{h} \lambda(2 \sqrt{2} b+3 \pi \lambda)}{16 b^{2}}\right] .
$$

Below $C^{*}$, a stronger intrinsic curvature $\kappa$ shortens the catastrophe time $\left\langle T_{c}\right\rangle$, by increasing the positive $m_{s^{-}}$ value, shifting the potential minimum and causing a lower energy barrier for lateral dissociation, while this effect is reversed above $C^{*}$ with $m_{s}$ turning negative. Figure 5(B) shows the frequency $1 /\left\langle T_{c}\right\rangle$ against $\kappa$ at varied concentrations (other parameters remain the same) to illustrate this phenomenon, with the estimated $L^{*} \approx 3$ (tubulin units) and $k_{a} C^{*} \approx 0.98$ (tubulins/sec) from Eq. 14.

The crossover $L^{*}$ may thus be defined as the minimum cap size to stabilize MT protofilament, as it cancels out the structural effect induced by the GDP-part to accel- erate catastrophe. Note that it has a different physical meaning from other cap models, where their minimum stabilizing cap is the boundary for the all-or-none catastrophe events. In contrast, even if the average GTP-cap in our model shrinks to this minimum size, the stochastic event of a catastrophe does not occur suddenly, and the protofilament still needs time to develop the crack to initiate catastrophe. The fact that the natural curvature $\kappa$ of GDP-units does not always facilitate catastrophe (it actually depends on the GTP-cap size) has not been stressed before.

\section{MT DYNAMICS UNDER COMPRESSIVE FORCE}

In vivo, MTs are known to be regulated by the force generated while it grows and pushes against barriers such as the cell periphery, resulting in an increased catastrophe frequency observed in experiment ${ }^{72}$.74. Here, we consider a constant compressive force $p$ applied on the protofilament tip with the cap size $L$. This force is assumed to propagate throughout the protofilament, while assumed not to cause the critical buckling of the whole MT filament. Under this scenario, we do not have to consider a more complicated case where $z_{0}$-direction no longer remains perpendicular to the filament axis before buckling, and the formulation of the Hamiltonian needs elaborate geometric discussion. Now the Hamiltonian for the protofilament upon compression $p$, under the assumption of small deflection $\underline{48 / 75} 77$, takes the form:

$$
\begin{gathered}
H_{p}=\int_{0}^{L} \frac{B}{2}\left(\frac{d^{2} z}{d s^{2}}\right)^{2}-\frac{p}{2}\left(\frac{d z}{d s}\right)^{2}+\frac{k}{2}(z-a)^{2} d s \\
+\int_{L}^{\infty} \frac{B}{2}\left(\frac{d^{2} z}{d s^{2}}-\kappa\right)^{2}-\frac{p}{2}\left(\frac{d z}{d s}\right)^{2}+\frac{k}{2}(z-a)^{2} d s,
\end{gathered}
$$

where the factor $-(d z / d s)^{2} / 2$ is the shrinkage in the length of the protofilament projected along the forcedirection, giving the mechanical work term. Following 
similar procedures (using the Rayleigh-Ritz method, and ignoring the boundary conditions at the interface between the cap and the GDP-part) to find out the effective potential as in Sec. 2.2 we first write down the forcebalance equation for a protofilament under compression $p$, based on the Hamiltonian of Eq. (15):

$$
B \frac{d^{4} z}{d s^{4}}-p \frac{d^{2} z}{d s^{2}}+k(z-a)=0 .
$$

The ansatz satisfying Eq. (16), with the key boundary conditions at ends: $z(0)=z_{0}, z(\infty)=0$, and $d z /\left.d s\right|_{s=\infty}=0$, is:

$$
z=a+\left[\left(z_{0}-a\right) \cos \left(\frac{s}{\lambda_{1}}\right)+A_{p} \sin \left(\frac{s}{\lambda_{1}}\right)\right] e^{\frac{-s}{\lambda_{2}}}
$$

with $\lambda_{1}=\frac{2}{\sqrt{2\left(\frac{k}{B}\right)^{\frac{1}{2}}+\frac{p}{B}}}$ and $\lambda_{2}=\frac{2}{\sqrt{2\left(\frac{k}{B}\right)^{\frac{1}{2}}-\frac{p}{B}}}$.

Unlike free-standing case, where we only have one characteristic length $\lambda$ for both decay and trigonometric functions in Eq. (8), the compressive force $p$ separates out these length scales, decreasing the wavelength $\lambda_{1}$, while increasing the decay length $\lambda_{2}$. Mathematically, in the standard way of solving this linear homogeneous equation, we assume the solution $e^{q s}$, with $q$ being a complex number, and insert it back into Eq. (16) to obtain a quartic equation: $B q^{4}+p q^{2}+k=0$. At $p=0$, the argument of the solution $q$ in the complex plane sits exactly at the angle $(2 n+1) \pi / 4$, where $n$ is an integer. These angles are irrelevant to the values of $k$ or $B$, thus only one length scale remaining when putting $q$ solution back into $e^{q s}$. On the other hand, with a non-zero $p$, the argument of the solution $q$ in the complex plane is no longer $(2 n+1) \pi / 4$, causing two length-scales - one for the real part of $q$ (decay function in $e^{q s}$ ) and the other for the imaginary part (trigonometric function). Physically, the decreased wavelength due to compression is to have as many wrinkles as possible along the protofilament, in order to minimize the bending penalty: a phenomenon similar to shortening of buckling wavelength with an increased force 48778 . As before, the amplitude $A_{p}$ is determined through energy minimization $d H_{p} / d A_{p}=0$, giving:

$$
A_{p}=\frac{-1}{\sqrt{4 B k-p^{2}}}\left\{p\left(z_{0}-a\right)+2 \kappa B\left[\cos \left(\frac{L}{\lambda_{1}}\right)-\frac{\sqrt{2 \sqrt{B k}-p}}{\sqrt{2 \sqrt{B k}+p}} \sin \left(\frac{L}{\lambda_{1}}\right)\right]\right\} .
$$

Equation (18) can be easily checked to reduce to the free-standing case of Eq. (9) at $p=0$. Now we insert the solution for the protofilament shape, Eq. (17) and the optimized amplitude $A_{p}$, back into the Hamiltonian functional of Eq. (19), and obtain the effective potential as a function of $z_{0}$. Once again, we obtain a quadratic form:

$$
V_{p}=\frac{k^{1 / 2}(\sqrt{B k}-p)}{2 \sqrt{2 \sqrt{B k}-p}}\left(z_{0}-a-m_{s p}\right)^{2}, \text { with } m_{s p}=\frac{\kappa B e^{-L / \lambda_{2}}}{\sqrt{B k}-p}\left[\cos \left(\frac{L}{\lambda_{1}}\right)+\sqrt{\frac{2 \sqrt{B k}-p}{2 \sqrt{B k}+p}} \sin \left(\frac{L}{\lambda_{1}}\right)\right]
$$

As expected, when $p=0$, the cosine- and sine-functions inside $m_{s p}$ can be merged to give a phase angle of $(-\pi / 4)$, recovering Eq. 10 of the force-free case. The compressive force $p$ decreases the restoring coefficient, and eventually flattens out the potential at $p_{c}=\sqrt{B k}$, known as the critical buckling force which initiates structural instability of the protofilament (we will not discuss the case beyond this critical value). In contrast, $\kappa$ has no effect on this restoring constant, as also observed in the freestanding case. It is noticeable that with the existence of the natural curvature $\kappa$, the shift of the potential minimum, $m_{s p}$, is increased as compression $p$ grows, lowering the energy barrier to break the lateral bonds. Yet, compression $p$ alone, without $\kappa$, cannot alter $m_{p s}$, and can only modify the shape of the effective potential.
Assuming that the potential minimum is not shifted past the bond-breaking distance (i. e. $m_{s p}<c-a$ ), the mean peel-off time for the tip to escape from this quadratic potential, known as the average catastrophe time in our model, is expressed as:

$$
\left\langle T_{c p}\right\rangle=\frac{\sqrt{2 \pi} \operatorname{Exp}\left[\frac{k^{1 / 2}(\sqrt{B k}-p)}{2 \sqrt{2 \sqrt{B k}-p}} \beta\left(c-a-m_{s p}\right)^{2}\right]}{D \beta^{3 / 2}\left(\frac{k^{1 / 2}(\sqrt{B k}-p)}{\sqrt{2 \sqrt{B k}-p}}\right)^{3 / 2}\left(c-a-m_{s p}\right)} .
$$

We plot in Fig. 6(A) the average catastrophe frequency, $1 /\left\langle T_{c p}\right\rangle$, using Eq. (20) and Eq. (4) for the steady-state $L$, against tubulin addition rate $\left(k_{g}\right.$-dependence is embedded in $m_{s p}$ through $L$ ) at several compressive forces 

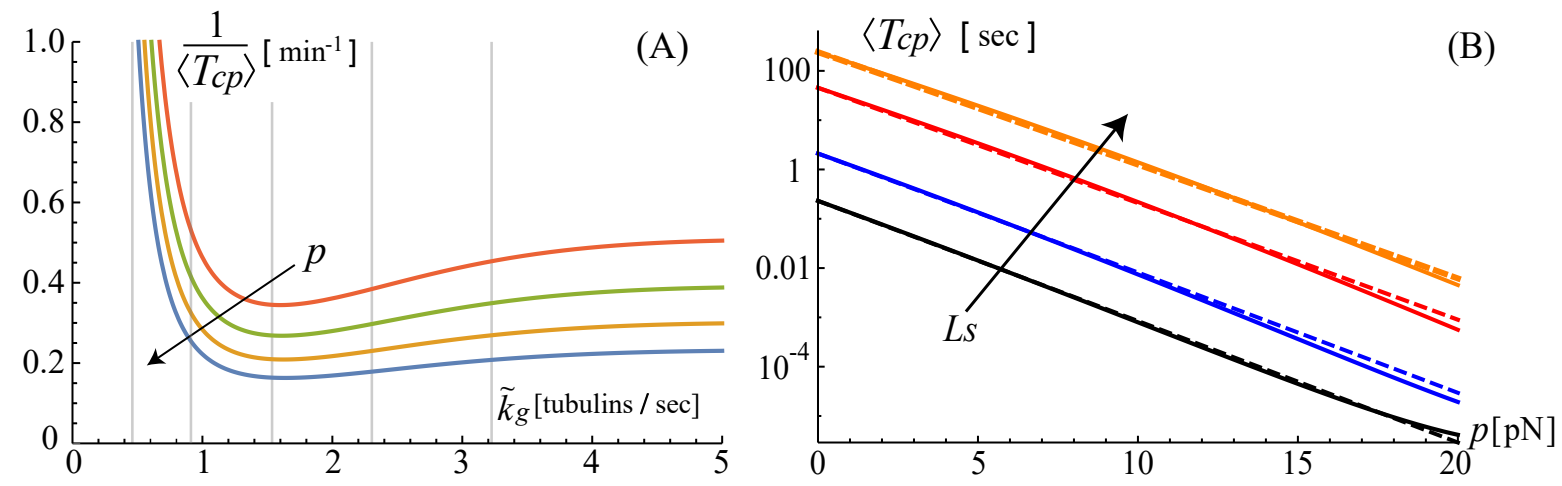

FIG. 6. (A) Catastrophe frequency $1 /\left\langle T_{c p}\right\rangle$ plotted against effective growth rate $\tilde{k}_{g}$, using Eq. 20, with compression $p=0$, $0.5,1.0$ and $1.5 \mathrm{pN}$, increased along the arrow. Grey vertical lines show the cap lengths $L_{s}=2,3$, 4 and 5 (tubulins), from left to right. (B) Mean catastrophe time $\left\langle T_{c p}\right\rangle$ plotted against compression $p$, while increasing the cap length $L_{s}$ from $0,1,2$ to 3 (tubulins) along the arrow. The solid lines are plotted by the exact expression, Eq. [20], while the dashed lines are from the approximation, Eq. 21.

$p$, with the same parameter values as defined in Fig. 4 . It can be clearly seen that compression $p$ accelerates the catastrophe process.

Equation 20 is still complicated enough to clearly expose the effect of compression $p$ in a more intuitive way, with $p$ appearing in the nontrivial function $m_{s p}$. However, in the weak-compression regime, $\left\langle T_{c p}\right\rangle$ may be approximated by a simple product of $\left\langle T_{c}\right\rangle$ and an exponential function that extracts this $p$-factor out:

$$
\begin{aligned}
& \frac{\left\langle T_{c p}\right\rangle}{\left\langle T_{c}\right\rangle} \approx\left(1+\frac{p}{\sqrt{B k}}\right) \operatorname{Exp}\left[\frac{-\beta k^{1 / 4}\left(c-a-m_{s}\right)^{2}}{2 \sqrt{2} B^{1 / 4}} f_{g} p\right] \\
& \text { with } f_{g}=\frac{3 c-3 a+5 m_{s}}{4\left(c-a-m_{s}\right)}
\end{aligned}
$$

$\left\langle T_{c}\right\rangle$ is the mean catastrophe time of the free-standing case, given in Eq. 12 or, equally, putting $p=0$ in Eq. 201. $m_{s}$ is the shift of the potential minimum without compression, obtained by letting $p=0$ in Eq. 19, and is a function of the steady-state cap size $L_{s}$.

The exponent in Eq. 21) highlights how the compressive force $p$ lowers the energy barrier to break the lateral bonds; its form is similar to the force-work term in the energy barrier for bond-breaking in the original potential, namely, $\left.V_{p}\right|_{z_{0}=c}$. There, we take the $p$-variable as zero in the denominator and in $m_{s p}$ of Eq. (19), while keeping only $p$ in the numerator as a crude estimation of force-induced energy decrease in this weakcompression case. We will then obtain the force-related term: $-p k^{1 / 4}\left(c-a-m_{s}\right)^{2} / 2 \sqrt{2} B^{1 / 4}$. The correction factor $f_{g}$ in Eq. 21) is to account for any possible $p$ contributions from $m_{s p}$ and the denominator in the original exact $V_{p}$ expression.

We plot in Fig. 6(B) the approximation for $\left\langle T_{c p}\right\rangle$ against the compressive force $p$, using Eq. (21) with varied cap lengths $L_{s}$, while other parameters remain the same as in Fig. 4. The plot clearly shows the approximation (21) matches very well with the exact $\left\langle T_{c p}\right\rangle$ values, even at medium-strong compression. But as $k$ and $B$ decrease, or $\kappa$ increases, the deviation from the exact $\left\langle T_{c p}\right\rangle$ would become more obvious. Note that the critical buckling force $p_{c}$ in this case is roughly $29.6 \mathrm{pN}$, estimated from $p_{c}=\sqrt{B k}$. However, we do not take compression $p$ all the way to this value, because our $\left\langle T_{c p}\right\rangle$ expression is derived by assuming $a+m_{s p}<c$ (namely, the position of the potential minimum does not exceed the bond-breaking point). Before $p$ reaches $p_{c}$, this assumption already no longer holds. Besides, our model does not account for the situation where the MT filament as a whole buckles. This critical buckling force $p_{M c}$ for the MT filament depends on its full length (in-vitro) ${ }^{\sqrt{75}}$, or on the bending rigidity and the elastic support from the cytoplasm (in vivo) ${ }^{75 / 77}$, giving a few $\mathrm{pN}$ for the former case, while larger than $100 \mathrm{pN}$ for the latter. This will result in a further cut-off at $p=p_{M c} / N$ in Fig. 6. where $N$ is the number of the protofilaments in this MT filament, and varies between 10 and 17 .

The $k_{a}$-constant embedded in $\tilde{k}_{g}$ may also depend on the force $p$. Previous studies 8183 described this dependence as $\tilde{k}_{a}=k_{a}^{0} \operatorname{Exp}(-\beta p \tilde{b})$, where $k_{a}^{0}$ is the addition rate constant without compressive forces and $\tilde{b}$ is the effective tubulin size. From the thermodynamic view, $\tilde{b}$ for a straight protofilament should be equal to the real tubulin size $b$, while for a curved protofilament, $\tilde{b}<b$. However, the experimentally-measured $\tilde{b}$-value was larger than $b$, the reason of which still remains unclear ${ }^{81}$. Equations (19), (20) and (21) are, in fact, general expression. To incorporate this $p$-effect on $k_{a}$, we simply need to write $k_{a}$, which is embedded in $\tilde{k}_{g}$ and $L_{s}$, explicitly in its $p$ relation. And it is expected that the compressive force $p$ will have a more pronounced effect than the case where $k_{a}$ is assumed irrelevant to $p$, as the force now shrinks the cap size qualitatively in an exponential way. 


\section{DISCUSSION}

We have explored the mean time for a microtubule to undergo a catastrophe, with and without a compressive force applied at the tip. Now we proceed to examine the probability distribution function of the stochastic time of individual catastrophe events. Many of the previous cap models, assuming a single tubulin unit for their stabilizing cap, would obtain a simple exponential decay function for this probability distribution - namely, the catastrophe is most likely to happen at short time ${ }^{26}-28 \mid 30$ ). This fails to explain the seemingly Gamma-like distribution function obtained in experiment $70|84| 85$ - catastrophe events are, in fact, rare at small times, and the probability distribution should go to zero as the reaction time is taken extremely small. As pointed out, this discrepancy may originate from the assumption of 'a single-step' mechanism for the MT catastrophe (hydrolyzing one GTP-unit at the tip) 30 . Adopting a multistep mechanism for the catastrophe, such as hydrolyzing more than two GTP-units in the cap $27 \mid 29$ or considering a multi-protofilament case ${ }^{30}$, could resolve this problem of non-zero catastrophe probability at very short time. Although we also adopt a 'single-step' mechanism (once the crack appears, the catastrophe takes place), our model does not suffer from this problem.

Our model of the tip escaping from the quadratic effective potential is essentially the Ornstein-Uhlenbeck process, of which the first-passage time distribution (FTPD) has been widely discussed ${ }^{49 \mid 50}$. Although there is no simple analytical expression for this FTPD throughout the entire time, the asymptotes for two extreme time-scales have elegant forms, allowing us to understand the trend of this distribution. We denote the probability distribution function for the catastrophe time as $f\left(T_{c}\right)$. At large times, $f\left(T_{c}\right)$ has an exponential trend 50 .

$$
f\left(T_{c}\right)=\frac{1}{\left\langle T_{c}\right\rangle} \operatorname{Exp}\left(\frac{-T_{c}}{\left\langle T_{c}\right\rangle}\right) .
$$

This asymptote for $T_{c}$ distribution is not a surprising result, indeed, it is the same as given in the single-step catastrophe mode 2627 . At short times, $f(t)$ has no explicit dependence on the mean first passage time $\left\langle T_{c}\right\rangle$, and it scales like

$$
f\left(T_{c}\right) \propto \beta D B^{1 / 4} k^{3 / 4} \operatorname{Exp}\left[\frac{-\left(c-a-m_{s}\right)^{2}}{2 D T_{c}}\right] .
$$

When $T_{c}$ is taken to zero, Eq. 23 would become zero. Therefore, we do not have non-zero possibility for the MT catastrophe at very short time.

\section{CONCLUSION}

In this paper, we take into account the structural effect of the GTP-cap of length $L$, and the natural curvature $\kappa$ of the GDP-part. We formulate the occurrence of the
MT catastrophe as the peel-off at the tip to produce a crack of one tubulin size in the lateral bonds, see Fig. 2 . The effective potential $V$ for the tip along this peel-off direction is obtained as a quadratic form of the tip height $z_{0}$, see Fig. 3, and is also a function of the cap size (which is regulated by the tubulin concentration $C$ ). We then apply the MFPT method to find the average rate for this peeling-off process, and obtain the mean catastrophe time $\left\langle T_{c}\right\rangle$. The result, Eq. 12 , exhibits roughly a double-exponential dependence of $C$, and is comparable with the experiment, see Fig. 4. In our model, we are also able to explicitly investigate the $\kappa$-effect on $\left\langle T_{c}\right\rangle-\kappa$ does not always facilitate the MT catastrophe, and there exists a cap size $L^{*}$ (and hence also a characteristic tubulin concentration $C^{*}$ ) defining the boundary of the reverse $\kappa$-effect, see Eq. (14) and Fig. 5. With the parameter values we find in Sec. 3, this characteristic size is estimated to be roughly three tubulin units. We also give critical comparisons with previous cap models for MT catastrophe, and discuss the plateau at high cap growth rates in our model, and the possible solutions to this issue.

We also consider the case with a constant compressive force $p$ on the protofilament tip. Following the similar procedures as in the free-standing case, the effective potential is also obtained as a quadratic function of $z_{0}$. The effect of the compressive force $p$ on the mean catastrophe time $\left\langle T_{c p}\right\rangle$ can approximately be extracted as an exponential-decay factor, together with a ratio $(1+p / \sqrt{B k})$ in the front, see Eq. (21). This result originates from the structural effect of $p$ on the protofilament as a whole, not simply due to the decrease in tubulin addition rate by compressive forces 8183 .

The real mechanism of MT catastrophe may be more complicated than the crack mechanism we explore in this paper. For example, the healing of the crack may be important at high tubulin concentrations 22 . Although, we did try to capture this effect in this paper, the resultant MT catastrophe curve decays too sharply with increasing cap growth rate (or equivalently, free tubulin concentration in solution). The MT catastrophe kinetics incorporating healing-effect may need a more careful treatment; MT catastrophe events are not simply governed by one protofilament (instead, it should be a consequence of collective dynamics of multiple protofilaments). We hope our model could be a first step toward understanding, in a more analytical way, the structural effect from the well-known existence of the straight GTP-cap and the intrinsic curvature of GDP-units on the catastrophe time. It is also very important for future studies to further elucidate the role of the crack, such as more accurate descriptions on the condition for MT catastrophe, in order to develop a more real mechanical (structural) model on the catastrophe kinetics. 


\section{Acknowledgements}

This work has been supported by the Theory of Condensed Matter Critical Mass Grant from EPSRC (EP/J017639).

${ }^{1}$ D. Chrétien and R. H. Wade, Biol. Cell 11, 161 (1991).

${ }^{2}$ R. Wade and D. ChrÃ@etien, J. Struct. Biol. 110, 1 (1993)

${ }^{3}$ T. Mitchison and M. Kirschner, Nature 312, 232 (1984)

${ }^{4}$ M. Kirschner and T. Mitchison, Cell 45, 329 (1986)

${ }^{5}$ T. Horio and H. Hotani, Nature 321, 605 (1986)

${ }^{6}$ R. A. Walker, E. T. O'Brien, N. K. Pryer, M. F. Soboeiro, W. A. Voter, H. P. Erickson, and E. D. Salmon, J. Cell Biol. 107, 1437 (1988).

'J. R. McIntosh and S. C. Landis, J. Cell Biol. 49, 468 (1971).

${ }^{8}$ P. Auckland and A. D. McAinsh, J. Cell Sci. 128, 3363 (2015)

${ }^{9}$ T. L. Hill, Proc. Natl. Acad. Sci. U.S.A. 82, 4404 (1985)

${ }^{10}$ T. E. Holy and S. Leibler, Proc. Natl. Acad. Sci. U.S.A. 91, 5682 (1994)

${ }^{11}$ R. Wollman, E. N. Cytrynbaum, J. T. Jones, T. Meyer, J. M. Scholey, and A. Mogilner, Curr. Biol. 15, 828 (2005)

${ }^{12}$ E. T. O'Brien, W. A. Voter, and H. P. Erickson, Biochemistry 26, 4148 (1987)

${ }^{15}$ A. A. Hyman, S. Salser, D. N. Drechsel, N. Unwin, and T. J. Mitchison, Mol. Biol. Cell 3, 1155 (1992)

${ }^{14}$ G. J. Brouhard and L. M. Rice, J. Cell Biol. 207, 323 (2014)

${ }^{15}$ R. Zhang, G. M. Alushin, A. Brown, and E. Nogales, Cell 162, 849 (2015).

${ }^{16}$ R. Melki, M. F. Carlier, D. Pantaloni, and S. N. Timasheff, Biochemistry 28, 9143 (1989)

${ }^{17}$ R. D. Vale, C. M. Coppin, F. J. Malik, F. amd Kull, and R. A. Milligan, J. Biol. Chem. 269, 23769 (1994).

${ }^{18}$ A. Grafmüller and G. A. Voth, Structure 19, 409 (2011)

${ }^{19}$ M. O. Steinmetz, K. R. A., W. Jahnke, K. N. Goldie, A. Lustig, and J. van Oostrum, EMBO J. 19, 572 (1989)

${ }^{20}$ I. M. Jánosi, D. Chrètien, and H. Flyvbjerg, Biophys. J. 83, 1317 (2002)

${ }^{21}$ V. VanBuren, L. Cassimeris, and D. J. Odde, Biophys. J. 89, $2911(2005)$

${ }^{22}$ P. Zakharov, N. Gudimchuk, A. Voevodin, V. an Tikhonravov, F. I. Ataullakhanov, and E. L. Grishchuk, Biophys. J. 109, 2574 (2015).

${ }^{23}$ D. N. Drechsel and M. W. Kirschner, Curr. Biol. 4, 1053 (1994)

${ }^{24} \mathrm{M}$. Caplow and J. Shanks, Mol. Biol. Cell 7, 663 (1996)

${ }^{25}$ T. Mitchison and M. Kirschner, Nature 312, 237 (1984)

${ }^{26}$ H. Flyvbjerg, T. E. Holy, and S. Leibler, Phys. Rev. Lett. 73, $2372(1994)$

${ }^{27}$ L. Brun, B. Rupp, J. J. Ward, and F. Nédélec, Proc. Natl. Acad. Sci. U. S. A. 106, 21173 (2009)

${ }^{28}$ P. Ranjith, D. Lacoste, K. Mallick, and J.-F. Joanny, Biophys. J. 96, 2146 (2009)

${ }^{29}$ P. Ranjith, A. B. Kolomeisky, and D. Lacoste, Biophys. J. 102, $1274(2012)$

${ }^{30}$ H. Bowne-Anderson, M. Zanic, M. Kauer, and J. Howard, Bioessays 35, 452 (2013)

${ }^{1}$ X. Li and A. B. Kolomeisky, J. Phys. Chem. B 117, 9217 (2013)

${ }^{32}$ V. Jemseena and M. Gopalakrishnan, Phys. Rev. E 88, 032717 (2013)

${ }^{33}$ H. P. Erickson and E. T. O'Brien, Annu. Rev. Biophys. Biomol. Struct. 21, 145 (1992)

${ }^{44} \mathrm{~J}$. Howard and A. A. Hyman, Nature 422, 753 (2003)

${ }^{35}$ A. Dimitrov, M. Quesnoit, S. Moutel, I. Cantaloube, C. Poüs, and F. Perez, Science 322, 1353 (2008)

${ }^{36}$ C. Tropini, E. A. Roth, M. Zanic, M. K. Gardner, and J. Howard, PLoS One 7, 1 (2012)

${ }^{37}$ M. K. Gardner, M. Zanic, and J. Howard, Curr. Opin. Cell Biol. 25, 14 (2013)

${ }^{38}$ T. Antal, P. L. Krapivsky, and S. Redner, J. Stat. Mech. Theory Exp. 2007, L05004 (2007)
${ }^{39}$ F.-A. Piedra, T. Kim, E. S. Garza, E. A. Geyer, A. Burns, X. Ye, and L. M. Rice, Mol. Biol. Cell 27, 3515 (2016)

${ }^{40}$ S. W. Manka and C. A. Moores, Nat. Struct. Mol. Biol. 25, 607 (2018)

${ }^{41}$ G. Margolin, H. V. Goodson, and M. S. Alber, Phys. Rev. E 83, 041905 (2011)

${ }^{42}$ G. Margolin, I. V. Gregoretti, T. M. Cickovski, C. Li, W. Shi, M. S. Alber, and H. V. Goodson, Mol. Biol. Cell 23, 642 (2012)

${ }^{43}$ C. Li, J. Li, H. V. Goodson, and M. S. Alber, Soft Matter 10, 2069 (2014)

${ }^{44}$ D. N. Drechsel, A. A. Hyman, M. H. Cobb, and M. W. Kirschner, Mol. Biol. Cell 3, 1141 (1992).

${ }^{45}$ A. Desai and T. J. Mitchison, Annu. Rev. Cell Dev. Biol. 13, 83 (1997)

${ }^{40}$ T. J. Itoh and H. Hotani, Cell Struct. Funct. 19, 279 (1994)

${ }^{47}$ Y. A. Komarova, A. S. Akhmanova, S. Kojima, N. Galjart, and G. G. Borisy, J. Cell Biol. 159, 589 (2002)

${ }^{48} \mathrm{~S}$. Timoshenko and J. M. Gere, Theory of elastic instability (McGraw-Hill, N. Y., 1961).

49 A. G. Nobile, L. M. Ricciardi, and L. Sacerdote, J. Appl. Probab. 22, 360 (1985)

${ }^{50}$ L. M. Ricciardi and S. Sato, J. Appl. Probab. 25, 43 (1988)

${ }^{51}$ P. Hänggi, P. Talkner, and M. Borkovec, Rev. Mod. Phys. 62, 251 (1990)

${ }^{52} \mathrm{H}$. Risken, The Fokker-Planck equation: methods of solution and applications (Springer, Berlin-Heidelberg, 1996).

${ }^{53}$ G. Hummer and A. Szabo, Biophys. J. 85, 5 (2003)

${ }^{54}$ H. T. Schek, M. K. Gardner, J. Cheng, D. J. Odde, and A. J. Hunt, Curr. Biol. 17, 1445 (2007)

${ }^{55}$ D. Seetapun, B. T. Castle, A. J. McIntyre, P. T. Tran, and D. J. Odde, Curr. Biol. 22, 1681 (2012)

${ }^{56}$ S. P. Maurer, F. J. Fourniol, G. Bohner, C. A. Moores, and T. Surrey, Cel 149, 371 (2012)

${ }^{57}$ D. S. Grebenkov, J. Phys. A 48, 013001 (2015)

${ }^{58}$ P. J. de Pablo, I. A. T. Schaap, F. C. Mackintosh, and C. F. Schmidt, Phys. Rev. Lett. 91, 098101 (2003)

${ }^{59}$ O. Kononova, Y. Kholodov, K. E. Theisen, K. A. Marx, R. I. Dima, F. I. Ataullakhanov, E. L. Grishchuk, and W. Barsegove, J. Am. Chem. Soc. 136, 17036 (2014).

${ }^{60}$ T. Müller-Reichert, D. Chrètien, F. Severin, and A. A. Hyman, Proc. Natl. Acad. Sci. U. S. A. 95, 3661 (1998)

${ }^{61}$ B. Gigant, P. A. Curmi, C. Martin-Babey, E. Charbautm, S. Lachkar, L. Lebeau, S. Siavoshian, A. Sobel, and M. Knossow, Cell 120, 809 (2000)

${ }^{62}$ B. Mickey and J. Howard, J. Cell Biol. 130, 909 (1995)

${ }^{63}$ V. VanBuren, D. J. Odde, and L. Cassimeris, Proc. Natl. Acad. Sci. U. S. A. 99, 6035 (2002)

${ }^{64}$ M. I. Molodtsov, E. L. Grishchuk, A. K. Efremov, J. R. McIntosh, and F. I. Ataullakhanov, Proc. Natl. Acad. Sci. U. S. A. 102, 4353 (2005)

${ }^{{ }^{5}}$ C. L. Asbury, J. F. Tien, and T. N. Davis, Trends Cell Biol. 21, 38 (2011)

${ }^{{ }^{6}}$ E. D. Salmon, W. M. Saxton, R. J. Leslie, M. L. Karow, and J. R. McIntosh, J. Cell Biol. 99, 2157 (1984).

${ }^{67}$ P. A. Deymier, Y. Yang, and J. Hoying, Phys. Rev. E 72, 021906 (2005)

${ }^{68}$ R. Melki, S. R. Fievez, and F.-F. Carlier, Biochemistry 35, 12038 (1996)

${ }^{{ }^{\circ}}$ A. Wu, E. Nogales, and J. Xing, Biophys. J. 102, 2687 (2012)

${ }^{70}$ M. K. Gardner, M. Zanic, C. Gell, V. Bormuth, and J. Howard, Cell 147, 1092 (2011)

${ }^{71}$ R. C. Weisenberg and W. J. Deery, Nature 263, 792 (1976)

${ }^{72}$ P. Tran, L. Marsh, V. Doye, S. Inoué, and F. Chang, J. Cell Biol. 153, 397 (2001)

${ }^{73}$ M. E. Janson, M. E. de Dood, and M. Dogterom, J. Cell Biol. 161, 1029 (2003)

${ }^{14}$ C. Tischer, D. Brunner, and M. Dogterom, Mol. Syst. Biol 5, $250(2009)$

${ }^{75}$ C. P. Brangwynne, F. C. MacKintosh, S. Kumar, N. A. Geisse, J. Talbot, L. Mahadevan, K. K. Parker, D. E. Ingber, and D. A. 
Weitz, J. Cell Biol. 173, 733 (2006)

${ }^{76}$ M. Das, A. J. Levine, and F. C. MacKintosh, Europhys. Lett. 84, 18003 (2008)

${ }^{\prime \prime}$ C.-T. Lee and E. M. Terentjev, J. Chem. Phys. 149, 145101 (2018)

${ }^{78}$ G. W. Hunt, M. K. Wadee, and N. N. Shiacolas, J. Appl. Mech. 60, 1033 (1993)

${ }^{r}$ S. H. Lee and A. M. Waas, Int. J. Nonlin. Mech. 25, 313 (1996)

${ }^{80}$ B. Audoly, Phys. Rev. E 84, 011605 (2011).
${ }^{81}$ M. Dogterom and B. Yurke, Science 278, 856 (1997)

${ }^{82}$ L. Laan, J. Husson, E. L. Munteanu, J. W. J. Kerssemakers, and M. Dogterom, Proc. Natl. Acad. Sci. U. S. A. 105, 8920 (2008) ${ }^{83}$ B. Zelinski and J. Kierfeld, Phys. Rev. E 87, 012703 (2013)

${ }^{84}$ D. Odde, L. Cassimeris, and H. M. Buettner, Biophys. J. 69, 796 (1995)

${ }^{85}$ A. Janulevicius, J. van Pelt, and A. van Ooyen, Biophys. J. 90, $788(2006)$ 